\section{Ultrasound-guided excision of nonpalpable malignant soft tissue tumors of the abdomen}

\section{Giovanni D Giannotti', Luay D Ailabouni' \& George I Salti†'}

'Department of Surgical Oncology, University of Illinois at Chicago, 840 S Wood St (M/C 820), Chicago, IL 60672, USA

†Author for correspondence: Tel.: +1 3129963288 = Fax: +1 3129969365 -geosalti@uic.edu

The use of intraoperative ultrasound to guide the excision of sonographically visible but nonpalpable lesions is a newer modality. Its use in intraoperative localization of recurrent malignant soft tissue tumors has not been reported. This report describes a technique of intraoperative localization by ultrasound to guide the excision of recurrent nonpalpable malignant soft tissue tumors of the abdominal wall in two patients. Tumors of both patients were successfully localized intraoperatively by ultrasonography and excised with adequate margins. This technique leads to reduced patient discomfort and anxiety, the avoidance of organizational requirements on the day of surgery, and allows for adequate margins of resection.

Intraoperative ultrasonography is increasingly being used to guide the excision of sonographically visible nonpalpable lesions. This is particularly true in cases of liver masses [1,2]. Its use has also been reported in localizing tumors of other sites such as breast [3-6], parathyroid [7] and pancreas [8]. Intraoperative ultrasonography also provides the operating surgeon with useful real-time diagnostic and staging information that may result in an alteration in the planned surgical approach. Current applications for intraoperative ultrasound include tumor staging, metastatic survey, guidance for metastasectomy and various tumor ablation procedures. Its use in intraoperative localization of recurrent malignant soft tissue tumors has not been reported.

\section{Patient one}

A 27-year-old obese Caucasian woman was referred with a large thoracoabdominal liposarcoma, diagnosed by means of percutaneous biopsy. A CT scan of the chest and abdomen revealed a tumor measuring $20 \times 30 \mathrm{~cm}$, involving the left chest wall, the left lower lobe of the lung, the diaphragm, extending to the abdominal cavity and almost occupying the left upper quadrant of the abdomen. She was treated with radical resection of the malignancy. Follow-up CT scan showed a new mass within the subcutaneous fat at the site of the surgical bed. This was not palpable but was visualized by ultrasound (Figure 1A) and proved to be a recurrent liposarcoma by image-guided core needle biopsy. The tumor was limited to the abdominal wall.

\section{Patient two}

A 40-year-old woman was referred with a diagnosis of recurrent left chest wall desmoid tumor noted on follow-up CT scan. The lesion was $9 \mathrm{~cm}$ in diameter and was located in the inframammary region to include the chest wall. We performed a chest wall resection to include portion of ribs 6,7 and 8. She had recovered well from that procedure. A follow-up MRI study demonstrated a $4-\mathrm{cm}$ mass on the lower portion of her previous resection area involving the left upper quadrant of the abdominal wall, but not involving intra-abdominal structures or chest wall including ribs. This was not palpable but was successfully localized with ultrasound (Figure 1B). An ultrasound-guided biopsy showed a recurrent desmoid tumor.

\section{Ultrasound-guided excision}

In both cases, intraoperative localization was performed with an ultrasound machine model number: GE E9 (GE healthcare, WI, USA) and a $12-5 \mathrm{MHz} 50$ and $38 \mathrm{~mm}$ linear array ultrasound probe.

The transducer was covered with a sterile sheath and sterile coupling gel was used during the surgical procedure. Localization was performed after the patient was prepared and draped for operation in a sterile fashion. The lesion image was first centered on the ultrasound screen with the probe in the transverse direction. A sterile marker was used to mark the skin at the two ends of the probe. A straight line was then drawn between these two marks and the process was repeated in the sagittal direction. Where the two lines intersected marked

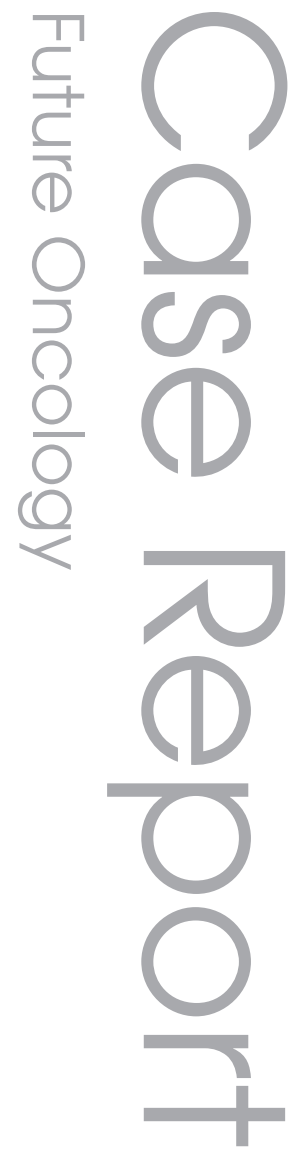

\section{Keywords}

- intraoperative ultrasound - localization

- soft tissue tumors

\section{future medicus $^{\text {pisg }}$}



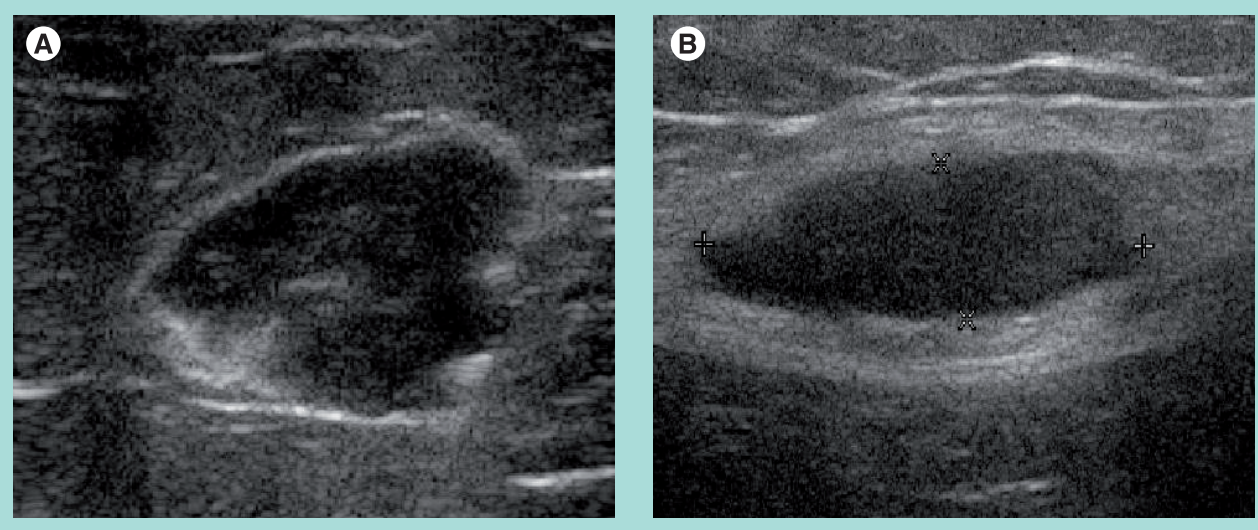

Figure 1. A 12-5 $\mathrm{MHz} 50-38 \mathrm{~mm}$ linear array ultrasound probe used intraoperatively to localize recurrent soft tissue tumors of the trunk. (A) Desmoid tumor. (B) Liposarcoma.

the center of the lesion. Accurate localization was reconfirmed by placing the ultrasound probe at that site and again visualizing the lesion on the screen.

Based on this localization, the tumor was marked and drawn on the skin. In addition, the planned surgical incision was marked on the skin to include a $2-3 \mathrm{~cm}$ margin.

\section{Surgical excision}

Once an incision was made, flaps of skin and subcutaneous tissue were raised to the predetermined margins. The ultrasound was used again in the open wound to confirm that the tumor was centered in the proposed area of excision. We then excised the tumor with predetermined margins. The excision was taken down deep enough to ensure a wide margin around the posterior aspect of the tumor. Specimen margins were marked and an immediate ultrasound of the specimen (in both transverse and sagittal planes) was performed. Postexcision specimen ultrasound was performed to confirm that the lesion was excised and that margins of excision were adequate. This was also confirmed histologically.

\section{Pathology \& outcome}

Patient one had a $1.5 \times 1.5 \mathrm{~cm}$ tumor excised with negative margins. The closest margin was $2 \mathrm{~cm}$. The patient developed distant metastasis without any evidence of local recurrence at 45 months and succumbed to disease 22 months later.

Patient two underwent excision of a $4.0 \times 1.5$ $\times 3.5 \mathrm{~cm}$ recurrent tumor with negative margins. The closest margin was $3 \mathrm{~cm}$; she remains disease-free at a 6-year follow-up.

\section{Discussion}

Ultrasound has gained popularity owing to its multiplanar use and lack of ionizing radiation. Traditionally, it has been most helpful differentiating solid from cystic lesions such as lesions within solid organs (i.e., liver and kidneys), and with palpable lesions such as in the breast. This modality has been criticized in its accuracy due to its operator dependence, and the presence of a learning curve [6]. In addition, the ease of portability of the ultrasound machine has facilitated its increasing use in the operating room offering the surgeon an intraoperative image of the lesion. We propose that this is a useful technique of intraoperative localization of nonpalpable malignant soft tissue tumors.

We found intraoperative ultrasound to be a safe and successful imaging modality in the localization of malignant soft tissue tumors of the trunk, which were otherwise nonpalpable. Similar to breast lesions, they were amenable to preoperative localization (to include wire placement). However, intraoperative ultrasonography offers improved patient comfort, decreased procedure time and eliminates the risk of wire displacement. In addition, assessments of margin status to achieve oncologically adequate margin of resection is of utmost importance. Our experience suggests that adequate and satisfactory surgical margins of nonpalpable soft tissue malignancies can be achieved using ultrasound guidance without the need for needle-wire placement. The surgeon is provided with a direct image of the lesion and its exact relationship to chosen boundaries of resection at any point during the procedure. Once the specimen is excised, specimen ultrasonography provides immediate confirmation 
that the tumor is confined within the specimen and that, grossly, no tumor remains in the specimen bed.

An important criterion for the ultrasoundguided technique is that the tumor must be readily imaged by ultrasound. The surgeon must have adequate knowledge and experience in performing ultrasound procedures. Alternatively, the surgeon may wish to involve a radiologist in the operating room. However, limited resources and coordination efforts may preclude this in many centers.

\section{Conclusion}

This study describes the successful use of intraoperative ultrasound to guide excision of nonpalpable recurrent malignant soft tissue tumors that would otherwise have to be localized preoperatively with an alternative technique such as placement of hookwire. It also leads to reduced patient discomfort and anxiety, the avoidance of organizational requirements on the day of surgery, and more importantly, allows for adequate margins of resection.

\section{Financial \& competing interests disclosure \\ The authors have no relevant affliations or financial involvement with any organization or entity with a financial interest in or financial conflict with the subject matter or materials discussed in the manuscript. This includes employment, consultancies, honoraria, stock ownership or options, expert testimony, grants or patents received or pending, or royalties. No writing assistance was utilized in the production of this manuscript.}

\section{Bibliography}

1. Sanatambrogio R, Opocher E, Ceretti AP et al:: Impact of intraoperative ultrasonographt in laparoscopic liver surgery. Surg. Endoscop. 21, 181-188 (2007).

2. Kruskal JB, Kane RA: Intraoperative US of the liver: techniques and clinical applications. Radiographics 26, 1067-1084 (2006).

3. Staren ED, O'Neil TP: Breast ultrasound. Surg. Clin. North Am. 78(2), 219-235 (1998).
4. Bennett IC, Greenslade J, Chiam H: Intraoperative ultrasound-guided excision of nonpalpable breast lesions. World J. Surg. 29, 369-374 (2005).

5. Harlow SP, Krag DN, Ames SE, Weaver DL: Intraoperative ultrasound localization to guide surgical excision of nonpalpable breast carcinoma. J. Am. Coll. Surg. 189, 241-246 (1999).

6. Rahusen FD, Bremers AJ, Fabry HF et al.: Ultrasound-guided lumpectomy of nonpalpable breast cancer versus wire-guided resection: a randomized clinical trial. Ann. Surg. Oncol. 9 , 994-998 (2002).

7. Kell MR, Sweeney KJ, Moran CJ et al: Minimally invasive parathyroidectomy with operative ultrasound localization of the adenoma. Surg. Endosc. 18, 1097-1098 (2004).

8. D'Onofrio M, Vecchiato F, Faccioli N et al.: Ultrasonography of the pancreas. 7. Intraoperative imaging. Abdom. Imaging 32(2), 200-206 (2007). 